\title{
The Proton Activation Data File PADF-2007
}

\author{
A.Yu. Konobeyev ${ }^{\text {a }, ~ C . H . M . ~ B r o e d e r s, ~ U . ~ F i s c h e r, ~ L . ~ M e r c a t a l i, ~ I . ~ S c h m u c k, ~ a n d ~ S . P . ~ S i m a k o v ~}$ \\ Institute for Reactor Safety, Forschungszentrum Karlsruhe, Postfach 3640, 76021 Karlsruhe, Germany
}

\begin{abstract}
To satisfy the increasing needs of nuclear data at intermediate energies the Proton Activation Data File (PADF-2007) has been generated. It contains 418,575 excitation functions of nuclear reactions for 2355 target nuclei from $\mathrm{Mg}$ to $\mathrm{Ra}$ at proton energies up to $150 \mathrm{MeV}$. The data are given for stable and unstable target nuclei including isomeric targets with half-lifes more than one second. The cross sections were obtained on the basis of model calculations using the TALYS and the ALICE/ASH codes and available experimental data.
\end{abstract}

\section{Introduction}

The evaluation and compilation of data for nuclear reactions induced by intermediate energy protons is important for a wide range of applications including the activation study for advanced nuclear systems, like ADS, and the study of production of radionuclides used in medicine and industry.

To satisfy the increasing needs in nuclear data at intermediate energies the Proton Activation Data File (PADF) has been developed. It contains calculated and evaluated excitation functions of nuclear reactions for target nuclei from $\mathrm{Mg}$ to $\mathrm{Ra}$ at proton energies up to $150 \mathrm{MeV}$. Data are given in PADF for stable and unstable target nuclei with half-life more than one second.

The cross sections were obtained on the basis of model calculations using the TALYS [1] and the ALICE/ASH codes [2] and existing experimental data.

As preparatory step, the work has been done to define nuclear models and approaches providing the best agreement with experimental nuclide yields. The comparison with experimental data has been made using nuclear models implemented in TALYS and ALICE/ASH. Approaches having minimal values of deviation factors [3] for the description of experimental data were applied for cross section calculations for PADF. Additional corrections have been made for calculated cross sections using available experimental data.

\section{Description of methods used to obtain cross sections}

\subsection{Calculations using nuclear models}

Calculations of cross sections for proton induced reactions including the radiative capture were performed using nuclear models implemented in the TALYS code and the ALICE/ASH code.

The use of TALYS involved calculations by the preequilibrium exciton model [4] and the Hauser-Feshbach

\footnotetext{
${ }^{a}$ Presenting author, e-mail: konobeev@irs.fzk.de
}

model. The pre-compound model implements new expressions for internal transition rates and new parameterization of the average squared matrix element for the residual interaction obtained using the optical model potential from ref. [5]. The particle-hole density is calculated taking into account the Pauli correction, the pairing correction and the final depth of nuclear potential well. The depth of the potential well has been parameterized as a function of the projectile energy and the mass of the target separately for incident neutrons and protons to reproduce the influence of surface effects on the first stage of interaction [1]. The multiple pre-equilibrium emission is considered up to arbitrary order of particle escape.

The phenomenological model from ref. [6] was used for the description of the pre-equilibrium complex particle emission from nuclei. The contribution of direct processes to the proton inelastic scattering was calculated using the ECIS code integrated in the TALYS code. The coupled channel model or DWBA was selected by TALYS for calculations using the available information about nuclear level schemes. The phenomenological model [1] was used to describe the giant resonance in the inelastic channel.

The geometry dependent hybrid model (GDH) [7] and the Weisskopf-Ewing model were applied for numerical calculations with the ALICE/ASH code. Intranuclear transition rates were calculated using the effective cross section of nucleonnucleon interactions in nuclear matter. Corrections were made to the GDH approach for the treatment of effects in peripheral nuclear regions $[2,8]$. The multiple precompound emission is described by an approximate approach [7]. The exciton state density is calculated taking into account pairing corrections, the correction for the Pauli principle and the final depth of the nuclear potential well for the exciton state $n=3$. The number of neutrons and protons for initial exciton state was calculated using realistic nucleon-nucleon interaction cross sections in nucleus [2].

The exciton coalescence pick-up model $[9,10]$ and the knock-out model [11] were used for the description of the preequilibrium complex particle emission. Parameters of models have been discussed in refs. [2,12].

The reaction cross section and transmission coefficients used in TALYS and ALICE/ASH calculations were obtained using optical model parameters from ref. [5]. 
Table 1. Values of deviation factors, equations (1)-(5) and $\mathrm{P}_{x}$, values, equation (6) obtained using (p,x) reaction cross sections calculated with the help of various codes and taken from various data libraries for target nuclei with the atomic number from 12 to 83 at the incident proton energy from 0 to $150 \mathrm{MeV}$. Number of experimental points is equal to 4125 . See explanations in the text.

\begin{tabular}{ccccccccc}
\hline $\begin{array}{c}\text { Deviation } \\
\text { factor }\end{array}$ & $\begin{array}{c}\text { TALYS- } \\
072\end{array}$ & $\begin{array}{c}\text { ALICE/ } \\
\text { ASH }\end{array}$ & DISCA-C & $\begin{array}{c}\text { Bertini/ } \\
\text { Dresner }\end{array}$ & $\begin{array}{c}\text { ENDF/B- } \\
\text { VI(8) }\end{array}$ & $\begin{array}{c}\text { ENDF/B- } \\
\text { VII }\end{array}$ & $\begin{array}{c}\text { JENDL- } \\
\text { HE }\end{array}$ & PADF \\
\hline $\mathrm{H}$ & 9.09 & 16.3 & 17.2 & 22.8 & 9.59 & 8.14 & 9.43 & 4.58 \\
$\mathrm{D}$ & 0.406 & 0.643 & 0.794 & 0.621 & 0.450 & 0.360 & 0.426 & 0.118 \\
$\mathrm{R}$ & 1.10 & 1.14 & 1.10 & 0.886 & 1.11 & 1.01 & 1.14 & 0.973 \\
$\mathrm{~F}$ & 1.78 & 3.68 & 4.62 & 11.3 & 1.79 & 1.74 & 1.74 & 1.29 \\
$\mathrm{~L}$ & 0.435 & 0.699 & 0.741 & 0.829 & 0.513 & 0.456 & 0.547 & 0.285 \\
$\mathrm{P}_{1.3}$ & 0.500 & 0.322 & 0.195 & 0.314 & 0.503 & 0.518 & 0.505 & 0.834 \\
$\mathrm{P}_{2.0}$ & 0.866 & 0.701 & 0.554 & 0.594 & 0.840 & 0.857 & 0.865 & 0.976 \\
$\mathrm{P}_{10.0}$ & 0.993 & 0.938 & 0.924 & 0.840 & 0.992 & 0.994 & 0.994 & 0.999 \\
\hline
\end{tabular}

The preparatory work comprised the comparison of available experimental radionuclide yields in neutron and proton induced reactions with calculations using TALYS and ALICE/ASH employing various nuclear models for the nuclear level density calculation.

Two modifications of the Fermi gas model [13] with different description of collective effects [1] and the microscopic approach of Goriely and coauthors [14] were used to obtain the nuclear level density in TALYS calculations. Calculations by the ALICE/ASH code were carried out using the Fermi gas model with the nuclear level density parameter A/9, the Fermi gas model [13] and the generalized superfluid nuclear model [15-17]. In the last case the asymptotic value of the nuclear level density parameter obtained using the RIPL data [17] is calculated as follows $\tilde{a} / \mathrm{A}=0.118-0.172 \mathrm{~A}^{-1 / 3}$ [30], which replaces the old systematics of the $\tilde{a}$-parameter [15].

More than seventeen thousands individual measurements of radionuclide yields in neutron induced reactions for target nuclei from $\mathrm{Al}$ to $\mathrm{Bi}$ at energies above $0.1 \mathrm{MeV}[18,19]$ and about nineteen thousands experimental points for proton induced reactions for targets from $\mathrm{Mg}$ to $\mathrm{Bi}$ at incident energies up to $150 \mathrm{MeV}$ [3] were used for the comparison with calculations.

The comparison $[3,18,19]$ shows a certain advantage of the Fermi gas model without explicit description of the collective enhancement [1] implemented in the TALYS code and the superfluid model [15-17] included in the ALICE/ASH code comparing with other approaches.

These two models for the calculation of the nuclear level density were used to obtain cross sections for the Proton Activation Data File.

The TALYS code was used to obtain reaction cross sections for stable nuclei and unstable nuclei with half-life more than ten minutes. Cross sections for targets with the shorter decay half-life were calculated by the ALICE/ASH code. Calculations for isomeric targets were performed using the TALYS code.

\subsection{Corrections using experimental data}

Experimental data available in EXFOR for proton induced reactions were used for the evaluation of nuclear reaction cross sections including the fitting and correction of calculated excitation functions.

Table 1 shows the result of the comparison of experimental data with cross sections calculated using various codes [20-22] and evaluated data taken from PADF, ENDF/B-VI, ENDF/B-VII and JENDL-HE. Deviation factors and $\mathrm{P}_{x}$ values shown in table 1 were calculated as follows $[18,19,23]$

$$
\begin{gathered}
H=\left(\frac{1}{N} \sum_{\mathrm{i}=1}^{N}\left(\frac{\sigma_{\mathrm{i}}^{\text {exp }}-\sigma_{\mathrm{i}}^{\text {calc }}}{\Delta \sigma_{\mathrm{i}}^{\exp }}\right)^{2}\right)^{1 / 2}, \\
D=\frac{1}{N} \sum_{\mathrm{i}=1}^{N}\left|\frac{\sigma_{\mathrm{i}}^{\text {exp }}-\sigma_{\mathrm{i}}^{\text {calc }}}{\sigma_{\mathrm{i}}^{\text {exp }}}\right|, \\
R=\frac{1}{N} \sum_{\mathrm{i}=1}^{N} \frac{\sigma_{\mathrm{i}}^{\text {calc }}}{\sigma_{\mathrm{i}}^{\text {exp }}}, \\
L=\left[\sum_{\mathrm{i}=1}^{N}\left(\frac{\sigma_{\mathrm{i}}^{\text {calc }}}{\Delta \sigma_{\mathrm{i}}^{\exp }}\right)^{2}\left(\frac{\sigma_{\mathrm{i}}^{\text {calc }}-\sigma_{\mathrm{i}}^{\text {exp }}}{\sigma_{\mathrm{i}}^{\text {calc }}}\right)^{2} / \sum_{\mathrm{i}=1}^{N}\left(\frac{\sigma_{\mathrm{i}}^{\mathrm{calc}}}{\Delta \sigma_{\mathrm{i}}^{\mathrm{exp}}\left[\log \left(\sigma_{\mathrm{i}}^{\text {exp }}\right)-\log \left(\sigma_{\mathrm{i}}^{\text {calc }}\right)\right]^{2}}\right)^{2}\right]^{1 / 2}, \\
P_{x}=N_{x} / N,
\end{gathered}
$$

where $\sigma_{\mathrm{i}}^{\exp }$ and $\Delta \sigma_{\mathrm{i}}^{\exp }$ are the measured cross section and its uncertainty, $\sigma_{\mathrm{i}}^{\text {calc }}$ is the calculated or evaluated cross section, $\mathrm{N}$ is the number of experimental points, $\mathrm{N}_{x}$ is the number of points with the ratio

$$
1 / x<\sigma_{\mathrm{i}}^{\text {calc }} / \sigma_{\mathrm{i}}^{\mathrm{exp}}<x .
$$

Figures 1-3 show examples of calculated cross sections and evaluated data from PADF. Some other examples are given in ref. [24].

\section{Content of PADF}

The Proton Activation Data File contains 418,575 excitation functions for nuclear reactions at proton energies up to $150 \mathrm{MeV}$. Data are available for 2355 stable target nuclei and 


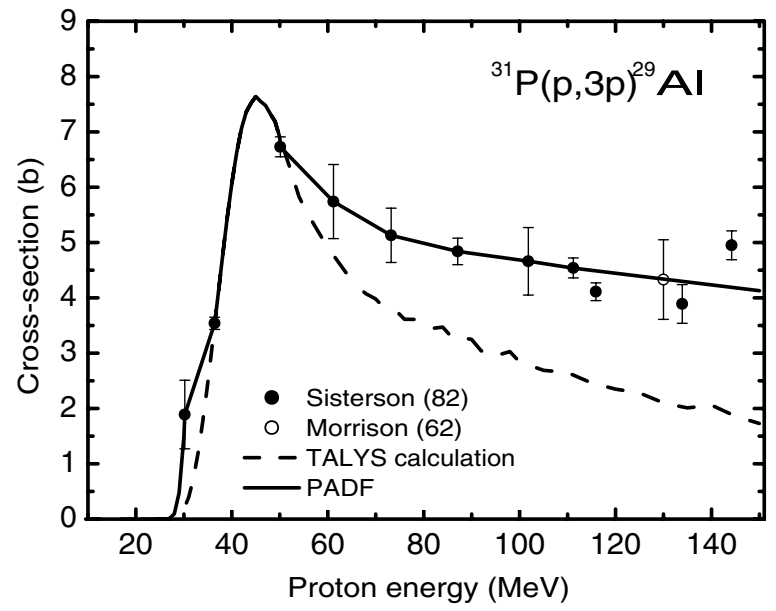

Fig. 1. Calculated and evaluated cross section for ${ }^{31} \mathrm{P}(\mathrm{p}, 3 \mathrm{p}){ }^{29} \mathrm{Al}$ reaction.

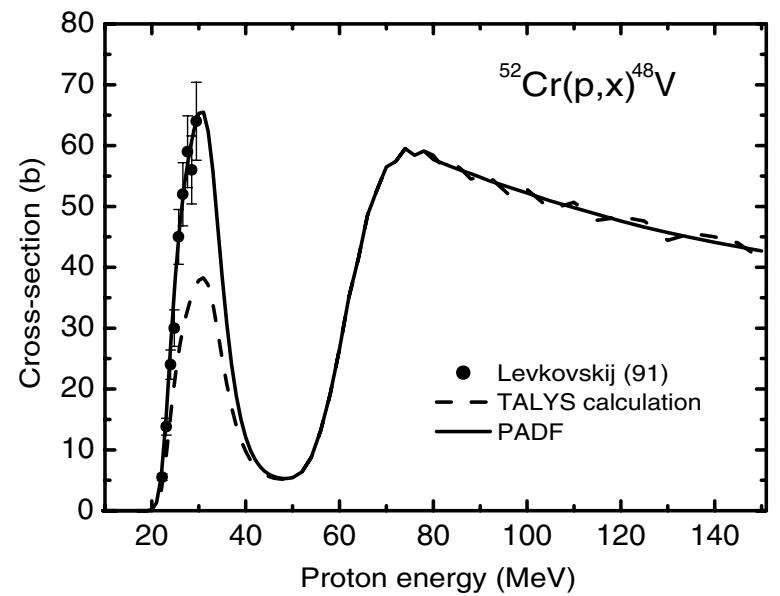

Fig. 2. Calculated and evaluated cross section for ${ }^{52} \mathrm{Cr}(\mathrm{p}, \mathrm{x}){ }^{48} \mathrm{~V}$ reaction.

unstable target nuclei with half-lifes $\left(\mathrm{T}_{1 / 2}\right)$ more than $1 \mathrm{sec}$ and with atomic number between 12 and 88 .

The calculation of cross sections for stable target nuclei and unstable nuclei with half-lifes more than 10 minutes was performed using the TALYS code. Cross sections for target nuclei with the decay half-life $1 \mathrm{sec}<\mathrm{T}_{1 / 2}<10 \mathrm{~min}$ were obtained using the ALICE/ASH code.

Calculations for isomeric targets with $\mathrm{T}_{1 / 2}>1$ hour were carried out by TALYS. Approximate data are included in the present version of PADF for other short-lived isomeric targets.

Isomeric residuals are presented in PADF for nuclei with $\mathrm{T}_{1 / 2}>1 \mathrm{sec}$.

Experimental cross sections available in EXFOR for proton induced reactions, $(\mathrm{p}, \mathrm{x})$ including $(\mathrm{p}, \gamma)$, were used for the correction of calculated excitation functions and the cross section evaluation. This involved cross sections for independent (non-cumulative) residual yields from 1434 EXFOR data sets. PADF includes also calculated neutron-, proton-, deuteron-, triton-, ${ }^{3} \mathrm{He}-, \alpha$-particle- and $\gamma$-production cross sections.

The excerpt of the PADF directory is shown in table 2.

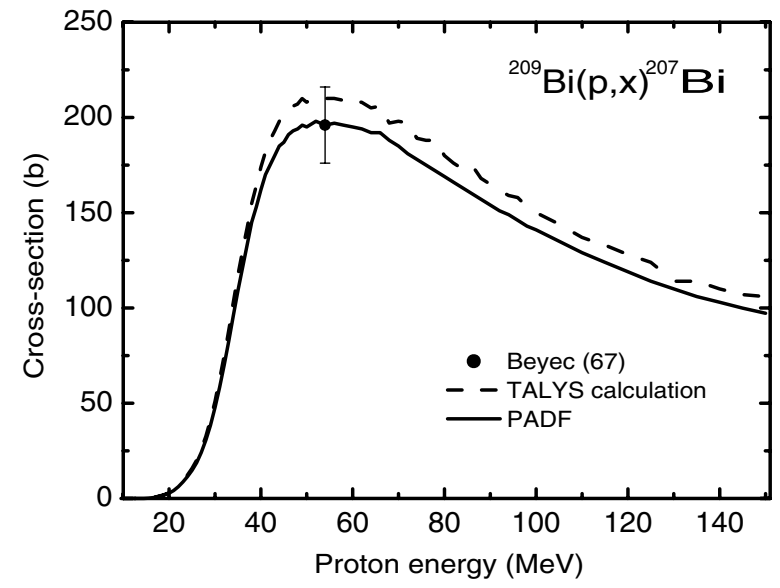

Fig. 3. Calculated and evaluated cross section for ${ }^{209} \mathrm{Bi}(\mathrm{p}, \mathrm{x}){ }^{207} \mathrm{Bi}$ reaction.

\section{Format of PADF}

PADF is available in pointwise and groupwise data format.

\subsection{Pointwise data format}

Data are presented in standard ENDF-6 format. Brief information about the method of the evaluation, nuclear models used for the calculation of cross sections and experimental data applied for the correction of calculated excitation functions is given in the section MT $=451$ of the file $\mathrm{MF}=1$.

The sum of all individual reaction cross sections is presented in $\mathrm{MF}=3, \mathrm{MT}=5$. The section MT $=5$ of the file $\mathrm{MF}=6$ contains yields of nuclei formed in nuclear reactions. The LIP variable indicates the state of the residual nucleus.

The number of neutron deficit isotopes for each element, included in PADF, does not permit the use of the common rule for material definition (MAT) [25]. In PADF, target nuclei are identified by MAT numbers taken from the radioactive data file JEFF-3.1/RDD file [26].

\subsection{Groupwise data format}

The groupwise format of PADF is the same as the one used for IEAF data [27].

The 256 group data files were generated using the NJOY code. The 175 group structure of VITAMIN-J was adopted at energies below $20 \mathrm{MeV}$. The energy bin of $1 \mathrm{MeV}$ was used for the energy range from 20 to $50 \mathrm{MeV}$ and $2 \mathrm{MeV}$ - bin for energies from 50 to $150 \mathrm{MeV}$.

\section{Conclusion}

Data file for the activation and transmutation analysis of materials irradiated with protons at energies up to $150 \mathrm{MeV}$ has been developed. It contains 418,575 evaluated excitation functions of $(\mathrm{p}, \mathrm{x})$ nuclear reactions for 2355 target nuclei from $\mathrm{Mg}$ to Ra. Data are available for stable and unstable target 
Table 2. The excerpt of the PADF directory. Values in brackets show the number of experimental work used for the cross section evaluation.

\begin{tabular}{lllr}
\hline Nucleus & half-life & origin & $\begin{array}{r}\text { number of } \\
\text { reactions }\end{array}$ \\
\hline$\ldots$ & & & \\
$26-\mathrm{Fe}-56$ & stable & TALYS, expr (10) & 99 \\
$26-\mathrm{Fe}-57$ & stable & TALYS, expr (5) & 101 \\
$26-\mathrm{Fe}-58$ & stable & TALYS, expr (5) & 100 \\
$26-\mathrm{Fe}-59$ & $44.50 \mathrm{~d}$ & TALYS & 109 \\
$26-\mathrm{Fe}-60$ & $1.501+$ 06 & TALYS & 105 \\
$26-\mathrm{Fe}-61$ & $5.980 \mathrm{~m}$ & ALICE/ASH & 169 \\
$26-\mathrm{Fe}-62$ & $1.133 \mathrm{~m}$ & ALICE/ASH & 171 \\
$26-\mathrm{Fe}-63$ & $6.100 \mathrm{~s}$ & ALICE/ASH & 178 \\
$26-\mathrm{Fe}-64$ & $2.000 \mathrm{~s}$ & ALICE/ASH & 182 \\
$26-\mathrm{Fe}-65$ & $1.300 \mathrm{~s}$ & ALICE/ASH & 188 \\
$27-\mathrm{Co}-54 \mathrm{~m} 1$ & $1.480 \mathrm{~m}$ & approximation & 127 \\
$27-\mathrm{Co}-55$ & $17.53 \mathrm{~h}$ & TALYS & 92 \\
$27-\mathrm{Co}-56$ & $77.31 \mathrm{~d}$ & TALYS & 97 \\
$27-\mathrm{Co}-57$ & $271.8 \mathrm{~d}$ & TALYS & 100 \\
$27-\mathrm{Co}-58$ & $70.86 \mathrm{~d}$ & TALYS & 102 \\
$27-\mathrm{Co}-58 \mathrm{~m} 1$ & $8.900 \mathrm{~h}$ & TALYS & 107 \\
$27-\mathrm{Co}-59$ & stable & TALYS, expr (21) & 103 \\
$27-\mathrm{Co}-60$ & $5.275 \mathrm{y}$ & TALYS & 105 \\
$27-\mathrm{Co}-60 \mathrm{~m} 1$ & $10.47 \mathrm{~m}$ & TALYS & 109 \\
$27-\mathrm{Co}-61$ & $1.650 \mathrm{~h}$ & TALYS & 107 \\
$27-\mathrm{Co}-62$ & $1.500 \mathrm{~m}$ & ALICE/ASH & 164 \\
\hline
\end{tabular}

nuclei including isomeric targets with half-lifes more that one second.

Cross sections for PADF have been obtained using results of calculations with the TALYS and the ALICE/ASH codes and available experimental data.

This work is supported in part by the European Commission through the IP EUROTRANS, the contract FI6W-CT-2004-516520.

\section{References}

1. A.J. Koning, S. Hilaire, M.C. Duijvestijn, NRG Report 21297/04.62741/P FAI/AK/AK, Dec 5, 2004.

2. C.H.M. Broeders, A.Yu. Konobeyev, Yu.A. Korovin, M. Blann, Forschungszentrum Karlsruhe Report FZKA 7183, 2006; http://bibliothek.fzk.de/zb/berichte/FZKA7183.pdf
3. L. Mercatali, A.Yu. Konobeyev, C.H.M. Broeders, On the uncertainty in the nuclear model calculation of neutron and proton induced reaction cross sections (these proceedings).

4. A.J. Koning, M.C. Duijvestijn, Nucl. Phys. A 744, 15 (2004).

5. A.J. Koning, J.P. Delaroche, Nucl. Phys. A 713, 231 (2003).

6. C.K. Kalbach Walker, PRECO-2000, March 2001; http://www. nndc.bnl.gov/nndescr/model-codes/preco-2000/index.html

7. M. Blann, H.K. Vonach, Phys. Rev. C 28, 1475 (1983).

8. Yu.A. Korovin, A.Yu. Konobeyev, P.E. Pereslavtsev, Progr. Nucl. Energy 40, 673 (2002).

9. A. Iwamoto, K. Harada, Phys. Rev. C 26, 1821 (1982).

10. K. Sato, A. Iwamoto, K. Harada, Phys. Rev. C 28, 1527 (1983).

11. A.Yu. Konobeyev, V.P. Lunev, Yu.N. Shubin, Acta Physica Slovaca 45, 705 (1995).

12. C.H.M. Broeders, A.Yu. Konobeyev, Nucl. Instrum. Meth. Phys. Res. B 234, 387 (2005).

13. A.V. Ignatyuk, G.N. Smirenkin, A.S. Tishin, Sov. J. Nucl. Phys. 21, 255 (1975).

14. S. Goriely, http://www-nds.iaea.org/RIPL-2/densities.html

15. A.V. Ignatyuk, K.K. Istekov, G.N. Smirenkin, Sov. J. Nucl. Phys. 29, 450 (1979).

16. A.V. Ignatyuk, R. Capote, IAEA-TECDOC-1506 (2006), p. 85; http://www-nds.iaea.org/RIPL-2/handbook.html

17. A.V. Ignatyuk, IAEA-TECDOC-1034 (1998), p. 65.

18. C.H.M. Broeders, A.Yu. Konobeyev, L. Mercatali, Kerntechnik 71, 174 (2006).

19. C.H.M. Broeders, A.Yu. Konobeyev, L. Mercatali, J. Nucl. Rad. Sci. 7, 1 (2006)

20. A.J. Koning, S. Hilaire, M.C. Duijvestijn, TALYS-0.72. Dec 21 2006; http://www.talys.eu/the-talys-team/arjan-koning/

21. C.H.M. Broeders, A.Yu. Konobeyev, Yu.A. Korovin, V.N. Sosnin, Forschungszentrum Karlsruhe Report FZKA 7221, June 2006, http://bibliothek.fzk.de/zb/berichte/FZKA7221.pdf

22. J.S. Hendricks, G.W. McKinney, J.W. Durkee et al., MCNPX, Version 26C, LA-UR-06-7991 Dec.7, 2006.

23. Yu.E. Titarenko (Project manager), ISTC 839B-99 (2001).

24. C.H.M. Broeders, U. Fischer, A.Yu. Konobeyev, L. Mercatali, S.P. Simakov, J. Nucl. Sci. Technol. (2007) (to be published).

25. M. Herman (ed.), BNL-NCS-44945-05-Rev. (2005).

26. A.J. Koning, O. Bersillon, R.A. Forrest et al., Proc. Int. Conf. on Nuclear Data for Science and Technology, Santa Fe, New Mexico, USA, 2004, p. 177.

27. U. Fischer, D. Leichtle, U. von Möllendorff, I. Schmuck, A.Yu. Konobeyev, Yu.A. Korovin, P.E. Pereslavtsev, Intermediate Energy Activation File IEAF-2001, NEA data bank Programme Library Package NEA-1656/01 (2001), RSICC Oak Ridge, DLC-217/IEAF-2001 (2002). 